\title{
Degenerate poly-Cauchy polynomials with a $q$ parameter
}

\section{Dae San Kim, Taekyun Kim ${ }^{2,3^{*}}$ and Dmitry V Dolgy ${ }^{4}$}

"Correspondence: tkkim@kw.ac.kr ${ }^{2}$ Department of Mathematics, Tianjin Polytechnic University, Tianjin, China

${ }^{3}$ Department of Mathematics, Kwangwoon University, Seoul, 139-701, S. Korea

Full list of author information is available at the end of the article

\begin{abstract}
In this paper, the degenerate poly-Cauchy polynomials with a $q$ parameter of the first and the second kind are introduced and their properties are studied. For these polynomials, some explicit formulas, recurrence relations, and connections with a few previously known families of polynomials are established.
\end{abstract}

MSC: 05A19; 05A40; 11B83

Keywords: degenerate poly-Cauchy polynomials with a $q$ parameter; umbral calculus

\section{Introduction}

Throughout the paper assume that $n, k \in \mathbb{Z}$ and $0 \neq q \in \mathbb{R}$. The poly-Cauchy polynomials with a $q$ parameter of the first kind $C_{n, q}^{(k)}(x)$ and of the second kind $\widehat{C}_{n, q}^{(k)}(x)$ are, respectively, defined by

$$
\begin{aligned}
& \operatorname{Lif}_{k}(\log (1+q t) / q)(1+q t)^{x / q}=\sum_{n \geq 0} C_{n, q}^{(k)}(x) \frac{t^{n}}{n !}, \\
& \operatorname{Lif}_{k}(-\log (1+q t) / q)(1+q t)^{-x / q}=\sum_{n \geq 0} \widehat{C}_{n, q}^{(k)}(x) \frac{t^{n}}{n !},
\end{aligned}
$$

for all $k \in \mathbb{Z}$, where

$$
\operatorname{Lif}_{k}(x)=\sum_{m \geq 0} \frac{x^{m}}{m !(m+1)^{k}}
$$

is the polylogarithm factorial function; see [1]. When $x=0, C_{n, q}^{(k)}=C_{n, q}^{(k)}(0)$, and $\widehat{C}_{n, q}^{(k)}=$ $\widehat{C}_{n, q}^{(k)}(0)$ are, respectively, called the poly-Cauchy numbers with a q parameter of the first kind and of the second kind. Note that $\operatorname{Lif}_{1}(x)=\frac{e^{x}-1}{x}$.

Here the degenerate versions are introduced for the poly-Cauchy polynomials with a $q$ parameter.

Definition 1.1 The degenerate poly-Cauchy polynomials with a q parameter of the first kind $C_{n, q}^{(k)}(\lambda, x)$ and of the second kind $\widehat{C}_{n, q}^{(k)}(\lambda, x)$ are, respectively, given by

(c) 2015 Kim et al. This article is distributed under the terms of the Creative Commons Attribution 4.0 International License (http://creativecommons.org/licenses/by/4.0/), which permits unrestricted use, distribution, and reproduction in any medium, provided you give appropriate credit to the original author(s) and the source, provide a link to the Creative Commons license, and indicate if changes were made. 


$$
\begin{aligned}
& \operatorname{Lif}_{k}\left(\frac{(1+q t)^{\lambda}-1}{q \lambda}\right)(1+q t)^{\frac{x}{q}}=\sum_{n \geq 0} C_{n, q}^{(k)}(\lambda, x) \frac{t^{n}}{n !}, \\
& \operatorname{Lif}_{k}\left(-\frac{(1+q t)^{\lambda}-1}{q \lambda}\right)(1+t)^{-\frac{x}{q}}=\sum_{n \geq 0} \widehat{C}_{n, q}^{(k)}(\lambda, x) \frac{t^{n}}{n !} .
\end{aligned}
$$

For $q=1, C_{n, 1}^{(k)}(\lambda, x)=C_{n}^{(k)}(\lambda, x)$ and $\widehat{C}_{n, 1}^{(k)}(\lambda, x)=\widehat{C}_{n}^{(k)}(\lambda, x)$ are the degenerate poly-Cauchy polynomials of the first kind and of the second kind, respectively, which are studied in [2]. When $x=0, C_{n, q}^{(k)}(\lambda, 0)$ and $\widehat{C}_{n, q}^{(k)}(\lambda, 0)$ are, respectively, called the degenerate poly-Cauchy numbers with a q parameter of the first kind and of the second kind.

In $[3,4]$, Carlitz introduced certain degenerate versions of Bernoulli and Euler polynomials. Almost half a century later these Carlitz degenerate Bernoulli polynomials were rediscovered under the name of Korobov polynomials of the second kind by Ustinov [5], while the degenerate version of the Bernoulli polynomials of the second kind were named the Korobov polynomials [6, 7]. It is remarkable that in recent years various degenerate versions of many important polynomials regained the attention of some researchers and many interesting results of them were obtained [2, 8-13]. Thus these have become an active area of research.

As was shown in the paper of Carlitz [3, 4], these degenerate versions have potential importance in number theory and combinatorics. For example, the authors have made some progress about symmetric identities involving the higher-order degenerate Euler and $q$-Euler polynomials by using the fermionic $p$-adic integrals. In a forthcoming paper, an investigation will be carried out as to some further results about the degenerate polyCauchy polynomials with a $q$ parameter which are of arithmetic and combinatorial nature.

The aim of this paper is to use umbral calculus techniques (see $[14,15])$ in order to derive some properties, recurrence relations, and identities for the degenerate poly-Cauchy polynomials with a $q$ parameter of the first kind and of the second kind.

From (1.2) and (1.3), one can see that $C_{n, q}^{(k)}(\lambda, x)$ is the Sheffer sequence for the pair $g(t)=\frac{1}{\operatorname{Lif}_{k}\left(\frac{e^{q \lambda t}-1}{q \lambda}\right)}, f(t)=\frac{e^{q t}-1}{q}$, and that $\widehat{C}_{n, q}^{(k)}(\lambda, x)$ is the Sheffer sequence for the pair $g(t)=$ $\frac{1}{\operatorname{Lif}_{k}\left(-\frac{e^{-q \lambda t}-1}{q \lambda}\right)}, f(t)=\frac{e^{-q t_{-}-1}}{q}$. Thus,

$$
C_{n, q}^{(k)}(\lambda, x) \sim\left(\frac{1}{\operatorname{Lif}_{k}\left(\frac{e^{q \lambda t}-1}{q \lambda}\right)}, \frac{e^{q t}-1}{q}\right), \quad \widehat{C}_{n, q}^{(k)}(\lambda, x) \sim\left(\frac{1}{\operatorname{Lif}_{k}\left(-\frac{e^{-q \lambda t}-1}{q \lambda}\right)}, \frac{e^{-q t}-1}{q}\right) .
$$

Umbral calculus has been used in numerous problems of mathematics and applied mathematics; for example, see $[2,16-28]$ and references therein.

\section{Explicit expressions}

Let us start by presenting several explicit formulas for the degenerate poly-Cauchy polynomials with a $q$ parameter, namely $C_{n, q}^{(k)}(\lambda, x)$ and $\widehat{C}_{n, q}^{(k)}(\lambda, x)$. To do so, recall here that Stirling numbers $S_{1}(n, k)$ of the first kind can be defined by means of exponential generating functions as

$$
\sum_{\ell \geq j} S_{1}(\ell, j) \frac{t^{\ell}}{\ell}=\frac{1}{j !} \log ^{j}(1+t),
$$


the Stirling numbers $S_{2}(n, k)$ of the second kind can be defined by the exponential generating functions as

$$
\sum_{n \geq k} S_{2}(n, k) \frac{x^{n}}{n !}=\frac{\left(e^{t}-1\right)^{k}}{k !}
$$

and can be defined by means of ordinary generating functions as

$$
(x \mid q)_{n}=q^{n}(x / q)_{n}=\sum_{m=0}^{n} S_{1}(n, m) q^{n-m} x^{m} \sim\left(1, \frac{e^{q t}-1}{q}\right),
$$

where $(x)_{n}=x(x-1)(x-2) \cdots(x-n+1)$ with $(x)_{0}=1$.

Theorem 2.1 For all $n \geq 0$,

$$
\begin{aligned}
& C_{n, q}^{(k)}(\lambda, x)=\sum_{j=0}^{n}\left(\sum_{\ell=j}^{n} \sum_{m=0}^{\ell-j} \frac{\left(\begin{array}{l}
\ell \\
j
\end{array}\right)}{(m+1)^{k}} S_{1}(n, \ell) S_{2}(\ell-j, m) q^{n-m-j} \lambda^{\ell-j-m}\right) x^{j}, \\
& \widehat{C}_{n, q}^{(k)}(\lambda, x)=\sum_{j=0}^{n}\left(\sum_{\ell=j}^{n} \sum_{m=0}^{\ell-j}(-1)^{m-j} \frac{\left(\begin{array}{l}
\ell \\
j
\end{array}\right)}{(m+1)^{k}} S_{1}(n, \ell) S_{2}(\ell-j, m) q^{n-m-j} \lambda^{\ell-j-m}\right) x^{j} .
\end{aligned}
$$

Proof By (1.4), one can see that

$$
\frac{1}{\operatorname{Lif}_{k}\left(\frac{e^{q \lambda t}-1}{q \lambda}\right)} C_{n, q}^{(k)}(\lambda, x) \sim\left(1, \frac{e^{q t}-1}{q}\right)
$$

Thus, by (2.3) and (2.2), one obtains

$$
\begin{aligned}
C_{n, q}^{(k)}(\lambda, x) & =\operatorname{Lif}_{k}\left(\frac{e^{q \lambda t}-1}{q \lambda}\right)(x \mid q)_{n}=\sum_{m=0}^{n} S_{1}(n, m) q^{n-m} \operatorname{Lif}_{k}\left(\frac{e^{q \lambda t}-1}{q \lambda}\right) x^{m} \\
& =\sum_{m=0}^{n} \sum_{\ell=0}^{m} S_{1}(n, m) q^{n-m} \frac{\left(e^{q \lambda t}-1\right)^{\ell}}{\ell !(\ell+1)^{k} \lambda^{\ell} q^{\ell}} x^{m} \\
& =\sum_{m=0}^{n} \sum_{\ell=0}^{m} \sum_{j=\ell}^{m} S_{1}(n, m) S_{2}(j, \ell) q^{n-m} \frac{\lambda^{j} q^{j}}{j !(\ell+1)^{k} \lambda^{\ell} q^{\ell}} t^{j} x^{m} \\
& =\sum_{m=0}^{n} \sum_{\ell=0}^{m} \sum_{j=\ell}^{m}\left(\begin{array}{l}
m \\
j
\end{array}\right) S_{1}(n, m) S_{2}(j, \ell) q^{n-m} \frac{\lambda^{j} q^{j}}{(\ell+1)^{k} \lambda^{\ell} q^{\ell}} x^{m-j} \\
& =\sum_{\ell=0}^{n} \sum_{m=0}^{\ell} \sum_{j=0}^{\ell-m}\left(\begin{array}{l}
\ell \\
j
\end{array}\right) S_{1}(n, \ell) S_{2}(\ell-j, m) q^{n-m-j} \lambda^{\ell-j-m} \frac{x^{j}}{(m+1)^{k}} \\
& =\sum_{j=0}^{n}\left(\sum_{\ell=j}^{n} \sum_{m=0}^{\ell-j} \frac{\left(\begin{array}{l}
\ell \\
j
\end{array}\right)}{(m+1)^{k}} S_{1}(n, \ell) S_{2}(\ell-j, m) q^{n-m-j} \lambda^{\ell-j-m}\right) x^{j},
\end{aligned}
$$

which completes the proof of the first formula. 
The second formula follows by similar arguments from the facts that

$$
\frac{1}{\operatorname{Lif}_{k}\left(-\frac{e^{-q \lambda t}-1}{q \lambda}\right)} \widehat{C}_{n, q}^{(k)}(\lambda, x) \sim\left(1, \frac{e^{-q t}-1}{q}\right)
$$

and $(-x \mid q)_{n}=\sum_{m=0}^{n}(-1)^{m} S_{1}(n, m) q^{n-m} x^{m} \sim\left(1, \frac{e^{-q t}-1}{q}\right)$.

Theorem 2.2 For all $n \geq 0$,

$$
\begin{aligned}
& C_{n, q}^{(k)}(\lambda, x)=\sum_{j=0}^{n}\left(\sum_{\ell=j}^{n} \sum_{m=0}^{\ell-j}\left(\begin{array}{l}
\ell \\
j
\end{array}\right) S_{1}(n, \ell) S_{2}(\ell-j, m) q^{n-m-j} C_{m, q}^{(k)}(\lambda, 0)\right) x^{j}, \\
& \widehat{C}_{n, q}^{(k)}(\lambda, x)=\sum_{j=0}^{n}\left(\sum_{\ell=j}^{n} \sum_{m=0}^{\ell-j}(-1)^{j}\left(\begin{array}{l}
\ell \\
j
\end{array}\right) S_{1}(n, \ell) S_{2}(\ell-j, m) q^{n-m-j} \widehat{C}_{m, q}^{(k)}(\lambda, 0)\right) x^{j} .
\end{aligned}
$$

Proof By (2.4) and (2.3), one has $C_{n, q}^{(k)}(\lambda, x)=\sum_{\ell=0}^{n} S_{1}(n, \ell) q^{n-\ell} \operatorname{Lif}_{k}\left(\frac{e^{q \lambda t}-1}{q \lambda}\right) x^{\ell}$. By (1.1), one obtains

$$
\begin{aligned}
C_{n, q}^{(k)}(\lambda, x) & =\left.\sum_{\ell=0}^{n} S_{1}(n, \ell) q^{n-\ell} \operatorname{Lif}_{k}\left(\frac{(1+q s)^{\lambda}-1}{q \lambda}\right)\right|_{s=\frac{q^{q t}-1}{q}} x^{\ell} \\
& =\sum_{\ell=0}^{n} \sum_{m=0}^{\ell} S_{1}(n, \ell) q^{n-\ell} C_{m, q}^{(k)}(\lambda, 0) \frac{\left(\frac{e^{q t}-1}{q}\right)^{m}}{m !} x^{\ell} .
\end{aligned}
$$

Thus, by (2.2), one gets

$$
\begin{aligned}
C_{n, q}^{(k)}(\lambda, x) & =\sum_{\ell=0}^{n} \sum_{m=0}^{\ell} \sum_{j=m}^{\ell} S_{1}(n, \ell) S_{2}(j, m) q^{n-\ell} C_{m, q}^{(k)}(\lambda, 0) \frac{q^{j-m}}{j !} t^{j} x^{\ell} \\
& =\sum_{\ell=0}^{n} \sum_{m=0}^{\ell} \sum_{j=m}^{\ell}\left(\begin{array}{l}
\ell \\
j
\end{array}\right) S_{1}(n, \ell) S_{2}(j, m) q^{n-\ell+j-m} C_{m, q}^{(k)}(\lambda, 0) x^{\ell-j} \\
& =\sum_{\ell=0}^{n} \sum_{m=0}^{\ell} \sum_{j=0}^{\ell-m}\left(\begin{array}{l}
\ell \\
j
\end{array}\right) S_{1}(n, \ell) S_{2}(\ell-j, m) q^{n-j-m} C_{m, q}^{(k)}(\lambda, 0) x^{j} \\
& =\sum_{j=0}^{n}\left(\sum_{\ell=j}^{n} \sum_{m=0}^{\ell-j}\left(\begin{array}{l}
\ell \\
j
\end{array}\right) S_{1}(n, \ell) S_{2}(\ell-j, m) q^{n-m-j} C_{m, q}^{(k)}(\lambda, 0)\right) x^{j},
\end{aligned}
$$

which completes the proof of the first formula.

For the second formula, one uses (2.5) to obtain

$$
\widehat{C}_{n, q}^{(k)}(\lambda, x)=\sum_{\ell=0}^{n}(-1)^{\ell} S_{1}(n, \ell) q^{n-\ell} \operatorname{Lif}_{k}\left(-\frac{e^{-q \lambda t}-1}{q \lambda}\right) x^{\ell} .
$$

Along the lines of the proof of the first formula, one derives 
Kim et al. Journal of Inequalities and Applications (2015) 2015:364

Page 5 of 15

$$
\begin{aligned}
\widehat{C}_{n, q}^{(k)}(\lambda, x) & =\sum_{\ell=0}^{n} \sum_{m=0}^{\ell} \sum_{j=m}^{\ell}(-1)^{\ell+j} S_{1}(n, \ell) S_{2}(j, m) q^{n-\ell} \widehat{C}_{m, q}^{(k)}(\lambda, 0) \frac{q^{j-m}}{j !} t^{j} x^{\ell} \\
& =\sum_{\ell=0}^{n} \sum_{m=0}^{\ell} \sum_{j=m}^{\ell}(-1)^{\ell+j}\left(\begin{array}{l}
\ell \\
j
\end{array}\right) S_{1}(n, \ell) S_{2}(j, m) q^{n-\ell+j-m} \widehat{C}_{m, q}^{(k)}(\lambda, 0) x^{\ell-j} \\
& =\sum_{\ell=0}^{n} \sum_{m=0}^{\ell} \sum_{j=0}^{\ell-m}(-1)^{j}\left(\begin{array}{l}
\ell \\
j
\end{array}\right) S_{1}(n, \ell) S_{2}(\ell-j, m) q^{n-j-m} \widehat{C}_{m, q}^{(k)}(\lambda, 0) x^{j} \\
& =\sum_{j=0}^{n}\left(\sum_{\ell=j}^{n} \sum_{m=0}^{\ell-j}(-1)^{j}\left(\begin{array}{l}
\ell \\
j
\end{array}\right) S_{1}(n, \ell) S_{2}(\ell-j, m) q^{n-m-j} \widehat{C}_{m, q}^{(k)}(\lambda, 0)\right) x^{j},
\end{aligned}
$$

as required.

Next, the transfer formula will be invoked. To do this, one observes that for any power series $g(t)=\sum_{m \geq 0} b_{m} \frac{t^{m}}{m !}, n \geq 0, a \neq 0$, and $p(x)=g(t) x^{n}, g(a t) x^{n}=a^{n} p(x / a)$. Recall that the Bernoulli polynomials $B_{n}^{(s)}(x)$ of order $s$ (see $[29,30]$ ) are defined by the generating function $\left(\frac{t}{e^{t}-1}\right)^{s} e^{x t}=\sum_{n \geq 0} B_{n}^{(s)}(x) \frac{t^{n}}{n !}$, or equivalently,

$$
B_{n}^{(s)}(x) \sim\left(\left(\frac{e^{t}-1}{t}\right)^{s}, t\right) .
$$

Theorem 2.3 For all $n \geq 1$,

$$
\begin{aligned}
C_{n, q}^{(k)}(\lambda, x)= & \sum_{j=0}^{n}\left(\sum_{\ell=0}^{n-j} \sum_{m=0}^{n-j-\ell}\left(\begin{array}{c}
n-1 \\
\ell
\end{array}\right)\left(\begin{array}{c}
n-\ell \\
j
\end{array}\right) S_{2}(n-\ell-j, m) \lambda^{n-\ell-j-m} q^{n-j-m} \frac{B_{\ell}^{(n)}}{(m+1)^{k}}\right) x^{j}, \\
\widehat{C}_{n, q}^{(k)}(\lambda, x)= & \sum_{j=0}^{n}\left(\sum_{\ell=0}^{n-j} \sum_{m=0}^{n-j-\ell}(-1)^{m-j}\left(\begin{array}{c}
n-1 \\
\ell
\end{array}\right)\left(\begin{array}{c}
n-\ell \\
j
\end{array}\right) S_{2}(n-\ell-j, m)\right. \\
& \left.\times \lambda^{n-\ell-j-m} q^{n-j-m} \frac{B_{\ell}^{(n)}}{(m+1)^{k}}\right) x^{j} .
\end{aligned}
$$

Proof By (2.4) and the fact that $x^{n} \sim(1, t)$, one obtains

$$
\frac{1}{\operatorname{Lif}_{k}\left(\frac{e^{q \lambda t}-1}{q \lambda}\right)} C_{n, q}^{(k)}(\lambda, x)=x\left(\frac{q t}{e^{q t}-1}\right)^{n} x^{-1} x^{n}=x\left(\frac{q t}{e^{q t}-1}\right)^{n} x^{n-1} .
$$

By (2.6), one gets

$$
\frac{1}{\operatorname{Lif}_{k}\left(\frac{e^{q \lambda t}-1}{q \lambda}\right)} C_{n, q}^{(k)}(\lambda, x)=x \sum_{\ell=0}^{n-1} B_{\ell}^{(n)} \frac{q^{\ell}}{\ell !} t^{\ell} x^{n-1}=\sum_{\ell=0}^{n-1}\left(\begin{array}{c}
n-1 \\
\ell
\end{array}\right) B_{\ell}^{(n)} q^{\ell} x^{n-\ell} .
$$

Thus, by (1.1) and (2.2), one has

$$
\begin{aligned}
C_{n, q}^{(k)}(\lambda, x) & =\sum_{\ell=0}^{n-1}\left(\begin{array}{c}
n-1 \\
\ell
\end{array}\right) B_{\ell}^{(n)} q^{\ell} \operatorname{Lif}_{k}\left(\frac{e^{q \lambda t}-1}{q \lambda}\right) x^{n-\ell} \\
& =\sum_{\ell=0}^{n-1} \sum_{m=0}^{n-\ell} \sum_{j=m}^{n-\ell}\left(\begin{array}{c}
n-1 \\
\ell
\end{array}\right)\left(\begin{array}{c}
n-\ell \\
j
\end{array}\right) S_{2}(j, m) B_{\ell}^{(n)} q^{\ell} \frac{(q \lambda)^{j}}{(m+1)^{k}(q \lambda)^{m}} x^{n-\ell-j}
\end{aligned}
$$




$$
\begin{aligned}
= & \sum_{\ell=0}^{n} \sum_{m=0}^{n-\ell} \sum_{j=0}^{n-\ell-m}\left(\begin{array}{c}
n-1 \\
\ell
\end{array}\right)\left(\begin{array}{c}
n-\ell \\
j
\end{array}\right) S_{2}(n-\ell-j, m) B_{\ell}^{(n)} q^{\ell} \frac{(q \lambda)^{n-\ell-j}}{(m+1)^{k}(q \lambda)^{m}} x^{j} \\
= & \sum_{j=0}^{n}\left(\sum_{\ell=0}^{n-j} \sum_{m=0}^{n-j-\ell}\left(\begin{array}{c}
n-1 \\
\ell
\end{array}\right)\left(\begin{array}{c}
n-\ell \\
j
\end{array}\right) S_{2}(n-\ell-j, m)\right. \\
& \left.\times \lambda^{n-\ell-j-m} q^{n-j-m} \frac{B_{\ell}^{(n)}}{(m+1)^{k}}\right) x^{j}
\end{aligned}
$$

which completes the proof of the first formula.

By using similar arguments to the above proof, using (2.5) instead (2.4), one derives the second formula.

Theorem 2.4 For all $n \geq 1$,

$$
\begin{aligned}
& C_{n, q}^{(k)}(\lambda, x)=\sum_{j=0}^{n}\left(\sum_{\ell=0}^{n-j} \sum_{m=0}^{n-j-\ell}\left(\begin{array}{c}
n-1 \\
\ell
\end{array}\right)\left(\begin{array}{c}
n-\ell \\
j
\end{array}\right) S_{2}(n-\ell-j, m) q^{n-m-j} B_{\ell}^{(n)} C_{m, q}^{(k)}(\lambda, 0)\right) x^{j}, \\
& \widehat{C}_{n, q}^{(k)}(\lambda, x)=\sum_{j=0}^{n}\left(\sum_{\ell=0}^{n-j} \sum_{m=0}^{n-j-\ell}(-1)^{j}\left(\begin{array}{c}
n-1 \\
\ell
\end{array}\right)\left(\begin{array}{c}
n-\ell \\
j
\end{array}\right) S_{2}(n-\ell-j, m) q^{n-m-j} B_{\ell}^{(n)} \widehat{C}_{m, q}^{(k)}(\lambda, 0)\right) x^{j} .
\end{aligned}
$$

Proof By using similar arguments to the proof of Theorem 2.2 together with (2.7) (or with the analog of (2.7) in the case of $\left.\widehat{C}_{n, q}^{(k)}(\lambda, x)\right)$, one obtains

$$
\begin{aligned}
C_{n, q}^{(k)}(\lambda, x) & =\sum_{\ell=0}^{n} \sum_{m=0}^{n-\ell} \sum_{j=0}^{n-\ell-m}\left(\begin{array}{c}
n-1 \\
\ell
\end{array}\right)\left(\begin{array}{c}
n-\ell \\
j
\end{array}\right) B_{\ell}^{(n)} S_{2}(n-\ell-j, m) q^{n-m-j} C_{m, q}^{(k)}(\lambda, 0) x^{j} \\
& =\sum_{j=0}^{n}\left(\sum_{\ell=0}^{n-j} \sum_{m=0}^{n-j-\ell}\left(\begin{array}{c}
n-1 \\
\ell
\end{array}\right)\left(\begin{array}{c}
n-\ell \\
j
\end{array}\right) S_{2}(n-\ell-j, m) q^{n-m-j} B_{\ell}^{(n)} C_{m, q}^{(k)}(\lambda, 0)\right) x^{j}
\end{aligned}
$$

and

$$
\begin{aligned}
\widehat{C}_{n, q}^{(k)}(\lambda, x) & =\sum_{\ell=0}^{n} \sum_{m=0}^{n-\ell} \sum_{j=0}^{n-\ell-m}(-1)^{j}\left(\begin{array}{c}
n-1 \\
\ell
\end{array}\right)\left(\begin{array}{c}
n-\ell \\
j
\end{array}\right) B_{\ell}^{(n)} S_{2}(n-\ell-j, m) q^{n-m-j} \widehat{C}_{m, q}^{(k)}(\lambda, 0) x^{j} \\
& =\sum_{j=0}^{n}\left(\sum_{\ell=0}^{n-j} \sum_{m=0}^{n-j-\ell}(-1)^{j}\left(\begin{array}{c}
n-1 \\
\ell
\end{array}\right)\left(\begin{array}{c}
n-\ell \\
j
\end{array}\right) S_{2}(n-\ell-j, m) q^{n-m-j} B_{\ell}^{(n)} \widehat{C}_{m, q}^{(k)}(\lambda, 0)\right) x^{j},
\end{aligned}
$$

which completes the proof.

Before proceeding recall here that the Bernoulli polynomials $b_{n}(x)$ (see [31]) of the second kind are defined by

$$
\frac{t}{\log (1+t)}(1+t)^{x}=\sum_{n \geq 0} C_{n}^{(1)}(x) \frac{x^{n}}{n !}=\sum_{n \geq 0} b_{n}(x) \frac{x^{n}}{n !}
$$


When $x=0, b_{n}=b_{n}(0)$ are called Bernoulli numbers of the second kind. With a $q$ parameter, one has

$$
\frac{q\left((1+q t)^{\frac{1}{q}}-1\right)}{\log (1+q t)}(1+t)^{x}=\sum_{n \geq 0} C_{n, q}^{(1)}(x) \frac{t^{n}}{n !}=\sum_{n \geq 0} C_{n, q}(x) \frac{t^{n}}{n !} .
$$

When $x=0$, we write $C_{n, q}=C_{n, q}(0)$. Also, it is well known (see [32]) that, for $k \geq 1$,

$$
\begin{aligned}
\operatorname{Lif}_{k}\left(\frac{\log (1+q t)}{q}\right) \\
=\frac{q}{\log (1+q t)} \underbrace{\int_{0}^{t} \frac{q}{(1+q t) \log (1+q t)} \cdots \int_{0}^{t} \frac{q}{(1+q t) \log (1+q t)}}_{(k-1) \text { times }}\left((1+q t)^{\frac{1}{q}}-1\right) d t \cdots d t .
\end{aligned}
$$

By induction on $k$, one has

$$
\operatorname{Lif}_{k}\left(\frac{\log (1+q t)}{q}\right)=\sum_{j_{1}, \ldots, j_{k} \geq 0} t^{j_{1}+\cdots+j_{k}} \frac{b_{j_{k}} q^{j_{k}}}{j_{k} !} \frac{C_{j_{1}, q}(-q)}{j_{1} !\left(j_{1}+1\right)} \prod_{i=2}^{k-1} \frac{b_{j_{i}}(-1) q^{j_{i}}}{j_{i} !\left(j_{1}+\cdots+j_{i}+1\right)}
$$

for all $k \geq 2$, and

$$
\operatorname{Lif}_{1}\left(\frac{\log (1+q t)}{q}\right)=\sum_{j_{1} \geq 0} C_{j_{1}, q} \frac{t^{j_{1}}}{j_{1} !}
$$

Thus, by changing variables, one obtains

$$
\begin{aligned}
& \operatorname{Lif}_{k}\left(\frac{(1+q t)^{\lambda}-1}{q \lambda}\right) \\
& \quad=\sum_{j_{1}, \ldots, j_{k} \geq 0}\left(\frac{e^{\frac{(1+q)^{\lambda}-1}{\lambda}}-1}{q}\right)^{j_{1}+\cdots+j_{k}} \frac{b_{j_{k}} q^{j_{k}}}{j_{k} !} \frac{C_{j_{1}, q}(-q)}{j_{1} !\left(j_{1}+1\right)} \prod_{i=2}^{k-1} \frac{b_{j_{i}}(-1) q^{j_{i}}}{j_{j} !\left(j_{1}+\cdots+j_{i}+1\right)},
\end{aligned}
$$

for all $k \geq 2$, and

$$
\operatorname{Lif}_{1}\left(\frac{(1+q t)^{\lambda}-1}{q \lambda}\right)=\sum_{j_{1} \geq 0} C_{j_{1}, q} \frac{\left(\frac{(1+q t)^{\lambda}-1}{\lambda}-1\right)^{j_{1}}}{j_{1} ! q^{j_{1}}} .
$$

Theorem 2.5 Let $n \geq 0$. Then

$$
\begin{aligned}
C_{n, q}^{(k)}(\lambda, x)= & \sum_{j_{1}+\cdots+j_{k} \leq n} \sum_{\ell=j_{1}+\cdots+j_{k}}^{n} \sum_{m=0}^{\ell}(-1)^{\ell-m} \frac{\left(\begin{array}{c}
\ell \\
\ell
\end{array}\right)}{\ell ! \lambda \ell} \frac{\left(j_{1}+\cdots+j_{k}\right) !}{q^{j_{1}+\cdots+j_{k}}} \frac{c_{j_{1}, q}(-q)}{j_{1} !\left(j_{1}+1\right)} \frac{b_{j_{k}} q^{j_{k}}}{j_{k} !} \\
& \times S_{2}\left(\ell, j_{1}+\cdots+j_{k}\right)(x+\lambda q m \mid q)_{n} \prod_{i=2}^{k-1} \frac{b_{j_{i}}(-1) q^{j_{i}}}{j_{i} !\left(j_{1}+\cdots+j_{i}+1\right)},
\end{aligned}
$$

for all $k \geq 2$, and

$$
C_{n, q}^{(1)}(\lambda, x)=\sum_{j_{1}=0}^{n} \sum_{\ell=j_{1}}^{n} \sum_{m=0}^{\ell}(-1)^{\ell-m} \frac{\left(\begin{array}{l}
\ell \\
m
\end{array}\right)}{\ell ! \lambda^{\ell}} \frac{C_{j_{1}, q}}{q^{j_{1}}} S_{2}\left(\ell, j_{1}\right)(x+\lambda q m \mid q)_{n} .
$$


Proof By (1.2), one has $C_{n, q}^{(k)}(\lambda, y)=\left\langle\operatorname{Lif}_{k}\left(\frac{(1+q t)^{\lambda}-1}{q \lambda}\right)(1+q t)^{\frac{y}{q}} \mid x^{n}\right\rangle$. Thus by (2.8), one gets

$$
\begin{aligned}
C_{n, q}^{(k)}(\lambda, y)= & \sum_{j_{1}+\cdots+j_{k} \leq n} \frac{c_{j_{1}, q}(-q)}{q^{j_{1}+\cdots+j_{k} j_{1} !\left(j_{1}+1\right)}} \frac{b_{j_{k}} q^{j_{k}}}{j_{k} !} \\
& \times \prod_{i=2}^{k-1} \frac{b_{j_{i}}(-1) q^{j_{i}}}{j_{i} !\left(j_{1}+\cdots+j_{i}+1\right)}\left\langle\left(e^{\frac{(1+q t)^{\lambda}-1}{\lambda}}-1\right)^{j_{1}+\cdots+j_{k}}(1+q t)^{\frac{y}{q}} \mid x^{n}\right\rangle,
\end{aligned}
$$

which, by (2.2), implies

$$
\begin{aligned}
C_{n, q}^{(k)}(\lambda, y) & \\
= & \sum_{j_{1}+\cdots+j_{k} \leq n} \frac{c_{j_{1}, q}(-q)}{q^{j_{1}+\cdots+j_{k}} j_{1} !\left(j_{1}+1\right)} \frac{b_{j_{k}} q^{j_{k}}}{j_{k} !} \prod_{i=2}^{k-1} \frac{b_{j_{i}}(-1) q^{j_{i}}}{j_{i} !\left(j_{1}+\cdots+j_{i}+1\right)} \\
& \times\left(j_{1}+\cdots+j_{k}\right) ! \sum_{\ell=j_{1}+\cdots+j_{k}}^{n} \frac{S_{2}\left(\ell, j_{1}+\cdots+j_{k}\right)}{\ell ! \lambda \ell}\left\langle\left((1+q t)^{\lambda}-1\right)^{\ell}(1+q t)^{\frac{y}{q}} \mid x^{n}\right\rangle \\
= & \sum_{j_{1}+\cdots+j_{k} \leq n} \frac{c_{j_{1}, q}(-q)}{q^{j_{1}+\cdots+j_{k} j_{1} !\left(j_{1}+1\right)}} \frac{b_{j_{k}} q^{j_{k}}}{j_{k} !} \prod_{i=2}^{k-1} \frac{b_{j_{i}}(-1) q^{j_{i}}}{j_{i} !\left(j_{1}+\cdots+j_{i}+1\right)} \\
& \times\left(j_{1}+\cdots+j_{k}\right) ! \sum_{\ell=j_{1}+\cdots+j_{k}}^{n} \sum_{m=0}^{\ell}\left(\begin{array}{l}
\ell \\
m
\end{array}\right)(-1)^{\ell-m} \frac{S_{2}\left(\ell, j_{1}+\cdots+j_{k}\right)}{\ell ! \lambda \ell}\left\langle(1+q t)^{\frac{y}{q}+\lambda m} \mid x^{n}\right\rangle .
\end{aligned}
$$

By using the fact that $\left\langle(1+q t)^{\frac{y}{q}+\lambda m} \mid x^{n}\right\rangle=(y+\lambda q m \mid q)_{n}$, the proof is completed for the case $k \geq 2$.

For $k=1$, by (1.2), one obtains

$$
C_{n, q}^{(1)}(\lambda, y)=\left\langle\operatorname{Lif}_{1}\left(\frac{(1+q t)^{\lambda}-1}{q \lambda}\right)(1+q t)^{\frac{y}{q}} \mid x^{n}\right\rangle=\sum_{j_{1}=0}^{n} \frac{C_{j_{1}, q}}{j_{1} ! q^{j_{1}}}\left\langle\left(e^{\frac{\left(1+q t t^{\lambda}-1\right.}{\lambda}}-1\right)^{j_{1}}(1+q t)^{\frac{y}{q}} \mid x^{n}\right\rangle
$$

which, by (2.2), implies

$$
\begin{aligned}
C_{n, q}^{(1)}(\lambda, x) & =\sum_{j_{1}=0}^{n} \sum_{\ell=j_{1}}^{m} \frac{C_{j_{1}, q} S_{2}\left(\ell, j_{1}\right)}{q^{j_{1}} \lambda^{\ell} \ell !}\left\langle\left((1+q t)^{\lambda}-1\right)^{\ell}(1+q t)^{\frac{y}{q}} \mid x^{n}\right\rangle \\
& =\sum_{j_{1}=0}^{n} \sum_{\ell=j_{1}}^{m} \sum_{m=0}^{\ell}\left(\begin{array}{c}
\ell \\
m
\end{array}\right)(-1)^{\ell-m} \frac{C_{j_{1}, q} S_{2}\left(\ell, j_{1}\right)}{q^{j_{1}} \lambda^{\ell} \ell !}\left\langle(1+q t)^{\frac{y}{q}+\lambda m} \mid x^{n}\right\rangle .
\end{aligned}
$$

By using the fact that $\left\langle(1+q t)^{\frac{y}{q}+\lambda m} \mid x^{n}\right\rangle=(y+\lambda q m \mid q)_{n}$, the proof is completed for the case $k=1$.

By similar arguments to the proof of Theorem 2.5 for the degenerate poly-Cauchy polynomials with a $q$ parameter of the first kind, one has the following result. 
Theorem 2.6 Let $n \geq 0$. Then

$$
\begin{gathered}
\widehat{C}_{n, q}^{(k)}(\lambda, x)=\sum_{j_{1}+\cdots+j_{k} \leq n} \sum_{\ell=j_{1}+\cdots+j_{k}}^{n} \sum_{m=0}^{\ell}(-1)^{\ell-m} \frac{\left(\begin{array}{l}
\ell \\
m
\end{array}\right)}{\ell !(-\lambda)^{\ell}} \frac{\left(j_{1}+\cdots+j_{k}\right) !}{q^{j_{1}+\cdots+j_{k}}} \frac{c_{j_{1}, q}(-q)}{j_{1} !\left(j_{1}+1\right)} \frac{b_{j_{k}} q^{j_{k}}}{j_{k} !} \\
\times S_{2}\left(\ell, j_{1}+\cdots+j_{k}\right)(\lambda q m-x \mid q)_{n} \prod_{i=2}^{k-1} \frac{b_{j_{i}}(-1) q^{j_{i}}}{j_{i} !\left(j_{1}+\cdots+j_{i}+1\right)},
\end{gathered}
$$

for all $k \geq 2$, and

$$
\widehat{C}_{n, q}^{(1)}(\lambda, x)=\sum_{j_{1}=0}^{n} \sum_{\ell=j_{1}}^{n} \sum_{m=0}^{\ell}(-1)^{\ell-m} \frac{\left(\begin{array}{l}
\ell \\
m
\end{array}\right)}{\ell !(-\lambda)^{\ell}} \frac{C_{j_{1}, q}}{q^{j_{1}}} S_{2}\left(\ell, j_{1}\right)(\lambda q m-x \mid q)_{n} .
$$

\section{Recurrences}

Note that the sequences of polynomials $C_{n, q}^{(k)}(\lambda, x)$ and $\widehat{C}_{n, q}^{(k)}(\lambda, x)$ are Sheffer sequences.

Thus they satisfy the Sheffer identity

$$
\begin{aligned}
& C_{n, q}^{(k)}(\lambda, x+y)=\sum_{j=0}^{n}\left(\begin{array}{l}
n \\
j
\end{array}\right) C_{j, q}^{(k)}(\lambda, x)(y \mid q)_{n-j}, \\
& \widehat{C}_{n, q}^{(k)}(\lambda, x+y)=\sum_{j=0}^{n}\left(\begin{array}{l}
n \\
j
\end{array}\right) \widehat{C}_{j, q}^{(k)}(\lambda, x)(-y \mid q)_{n-j} .
\end{aligned}
$$

Next, one shows several recurrences for the sequence of poly-Cauchy polynomials with a $q$ parameter of the first kind and of the second kind.

Theorem 3.1 For all $n \geq 1$,

$$
C_{n, q}^{(k)}(\lambda, x+q)=C_{n, q}^{(k)}(\lambda, x)+n q C_{n-1, q}^{(k)}(\lambda, x), \quad \widehat{C}_{n, q}^{(k)}(\lambda, x-q)=\widehat{C}_{n, q}^{(k)}(\lambda, x)+n q \widehat{C}_{n-1, q}^{(k)}(\lambda, x) .
$$

Proof Note that $f(t) S_{n}(x)=n S_{n-1}(x)$ for any $S_{n}(x) \sim(g(t), f(t))$ (see [14, 15]). Hence, by (1.4), one has

$$
\frac{e^{q t}-1}{q} C_{n, q}^{(k)}(\lambda, x)=n C_{n-1, q}^{(k)}(\lambda, x), \quad \frac{e^{-q t}-1}{q} \widehat{C}_{n, q}^{(k)}(\lambda, x)=n \widehat{C}_{n-1, q}^{(k)}(\lambda, x),
$$

which implies

$$
C_{n, q}^{(k)}(\lambda, x+q)=C_{n, q}^{(k)}(\lambda, x)+n q C_{n-1, q}^{(k)}(\lambda, x), \quad \widehat{C}_{n, q}^{(k)}(\lambda, x-q)=\widehat{C}_{n, q}^{(k)}(\lambda, x)+n q \widehat{C}_{n-1, q}^{(k)}(\lambda, x),
$$

as required.

Theorem 3.2 For $n \geq 0$,

$$
\begin{aligned}
C_{n+1 ; q}^{(k)}(\lambda, x)= & x C_{n, q}^{(k)}(\lambda, x-q)-\sum_{m=0}^{n} \sum_{\ell=0}^{m+1} \sum_{j=0}^{m+1-\ell} \frac{\left(\begin{array}{c}
m+1 \\
j
\end{array}\right)}{m+1} \lambda^{j} q^{n-\ell+1} S_{1}(n, m) \\
& \times S_{2}(m+1-j, \ell) d_{\ell, q}^{(k)}(\lambda) B_{j}\left(\frac{x+(\lambda-1) q}{q \lambda}\right)
\end{aligned}
$$




$$
\begin{aligned}
\widehat{C}_{n+1 ; q}^{(k)}(\lambda, x)= & -x \widehat{C}_{n, q}^{(k)}(\lambda, x+q)-\sum_{m=0}^{n} \sum_{\ell=0}^{m+1} \sum_{j=0}^{m+1-\ell} \frac{\left(\begin{array}{c}
m+1 \\
j
\end{array}\right)}{m+1} \lambda^{j} q^{n-\ell+1} S_{1}(n, m) \\
& \times S_{2}(m+1-j, \ell) \widehat{d}_{\ell, q}^{(k)}(\lambda) B_{j}\left(\frac{-x+(\lambda-1) q}{q \lambda}\right)
\end{aligned}
$$

where $d_{\ell, q}^{(k)}(\lambda)=C_{\ell, q}^{(k)}(\lambda, 0)-C_{\ell, q}^{(k-1)}(\lambda, 0)$ and $\widehat{d}_{\ell, q}^{(k)}(\lambda)=\widehat{C}_{\ell, q}^{(k)}(\lambda, 0)-\widehat{C}_{\ell, q}^{(k-1)}(\lambda, 0)$.

Proof Recall that

$$
\left(\operatorname{Lif}_{k}(x)\right)^{\prime}=\frac{\operatorname{Lif}_{k-1}(x)-\operatorname{Lif}_{k}(x)}{x},
$$

and $S_{n+1}(x)=\left(x-\frac{g^{\prime}(t)}{g(t)}\right) \frac{1}{f^{\prime}(t)} S_{n}(x)$ for any $S_{n}(x) \sim(g(t), f(t))$ (see [14, 15]). Thus, in the case of (1.4), one obtains

$$
C_{n+1 ; q}^{(k)}(\lambda, x)=x C_{n, q}^{(k)}(\lambda, x-q)-e^{-q t} \frac{g^{\prime}(t)}{g(t)} C_{n, q}^{(k)}(\lambda, x),
$$

where $g(t)=\frac{1}{\operatorname{Lif}_{k}\left(\frac{q q^{\lambda \lambda t}-1}{q \lambda}\right)}$. Note that $\frac{g^{\prime}(t)}{g(t)}=(\log (g(t)))^{\prime}=-\left(\log \operatorname{Lif}_{k}\left(\frac{e^{q \lambda t}-1}{q \lambda}\right)\right)^{\prime}$, which leads to

$$
\frac{g^{\prime}(t)}{g(t)}=\frac{-1}{\operatorname{Lif}_{k}\left(\frac{e^{q \lambda t}-1}{q \lambda}\right)}\left(\operatorname{Lif}_{k-1}\left(\frac{e^{q \lambda t}-1}{q \lambda}\right)-\operatorname{Lif}_{k}\left(\frac{e^{q \lambda t}-1}{q \lambda}\right)\right) \frac{\lambda q e^{\lambda q t}}{e^{\lambda q t}-1}
$$

Thus,

$$
e^{-q t} \frac{g^{\prime}(t)}{g(t)} C_{n ; q}^{(k)}(\lambda, x)=\frac{1}{t}\left(A_{k}-A_{k-1}\right) \frac{1}{\operatorname{Lif}_{k}\left(\frac{e^{q \lambda \lambda}-1}{q \lambda}\right)} C_{n, q}^{(k)}(\lambda, x),
$$

where $A_{k}-A_{k-1}=\frac{\lambda q t e^{(\lambda-1) q t}}{e^{\lambda q t}-1} \operatorname{Lif}_{k}\left(\frac{e^{q \lambda t}-1}{q \lambda}\right)-\frac{\lambda q t^{(\lambda-1) q t}}{e^{\lambda q t}-1} \operatorname{Lif}_{k-1}\left(\frac{e^{q \lambda t}-1}{q \lambda}\right)$ has order at least one (the order of a non-zero power series $f(t)$ is the smallest integer $k$ for which the coefficient of $t^{k}$ in $f(t)$ does not vanish). So, by the fact that $\frac{1}{\operatorname{Lif}_{k}\left(\frac{q^{\lambda \lambda t}-1}{q \lambda}\right)} C_{n, q}^{(k)}(\lambda, x)=(x \mid q)_{n}=$ $\sum_{m=0}^{n} S_{1}(n, m) q^{n-m} x^{n}$ (see (2.3)), one has

$$
\begin{aligned}
e^{-q t} \frac{g^{\prime}(t)}{g(t)} C_{n ; q}^{(k)}(\lambda, x) & =\sum_{m=0}^{n} S_{1}(n, m) q^{n-m} \frac{1}{t}\left(A_{k}-A_{k-1}\right) x^{m} \\
& =\sum_{m=0}^{n} \frac{S_{1}(n, m)}{m+1} q^{n-m}\left(A_{k}-A_{k-1}\right) x^{m+1} .
\end{aligned}
$$

On the other hand, by (2.2), one gets

$$
\begin{aligned}
A_{k} x^{m+1} & =\left.\frac{\lambda q t e^{(\lambda-1) q t}}{e^{\lambda q t}-1} \operatorname{Lif}_{k}\left(\frac{(1+q s)^{\lambda}-1}{q \lambda}\right)\right|_{s=\frac{q t_{-1}}{q}} x^{m+1} \\
& =\frac{\lambda q t e^{(\lambda-1) q t}}{e^{\lambda q t}-1} \sum_{\ell=0}^{m+1} C_{\ell, q}^{(k)}(\lambda, 0) \frac{\left(e^{q t}-1\right)^{\ell}}{\ell ! q^{\ell}} x^{m+1}
\end{aligned}
$$




$$
\begin{aligned}
& =\frac{\lambda q t e^{(\lambda-1) q t}}{e^{\lambda q t}-1} \sum_{\ell=0}^{m+1} \sum_{j=\ell}^{m+1} C_{\ell, q}^{(k)}(\lambda, 0) S_{2}(j, \ell) q^{-\ell} \frac{(q t)^{j}}{j !} x^{m+1} \\
& =\sum_{\ell=0}^{m+1} \sum_{j=\ell}^{m+1} C_{\ell, q}^{(k)}(\lambda, 0) S_{2}(j, \ell) q^{j-\ell}\left(\begin{array}{c}
m+1 \\
j
\end{array}\right) \frac{\lambda q t e^{(\lambda-1) q t}}{e^{\lambda q t}-1} x^{m+1-j} \\
& =\sum_{\ell=0}^{m+1} \sum_{j=\ell}^{m+1} C_{\ell, q}^{(k)}(\lambda, 0) S_{2}(j, \ell) q^{j-\ell}\left(\begin{array}{c}
m+1 \\
j
\end{array}\right)(\lambda q)^{m+1-j} B_{m+1-j}\left(\frac{x+(\lambda-1) q}{q \lambda}\right) \\
& =\sum_{\ell=0}^{m+1} \sum_{j=0}^{m+1-\ell}\left(\begin{array}{c}
m+1 \\
j
\end{array}\right) \lambda^{j} q^{m-\ell+1} C_{\ell, q}^{(k)}(\lambda, 0) S_{2}(m+1-j, \ell) B_{j}\left(\frac{x+(\lambda-1) q}{q \lambda}\right) .
\end{aligned}
$$

Hence,

$$
\begin{aligned}
C_{n+1 ; q}^{(k)}(\lambda, x)= & x C_{n, q}^{(k)}(\lambda, x-q)-\sum_{m=0}^{n} \sum_{\ell=0}^{m+1} \sum_{j=0}^{m+1-\ell} \frac{\left(\begin{array}{c}
m+1 \\
j
\end{array}\right)}{m+1} \lambda^{j} q^{n-\ell+1} S_{1}(n, m) \\
& \times S_{2}(m+1-j, \ell) d_{\ell, q}^{(k)}(\lambda) B_{j}\left(\frac{x+(\lambda-1) q}{q \lambda}\right)
\end{aligned}
$$

where $d_{\ell, q}^{(k)}(\lambda)=C_{\ell, q}^{(k)}(\lambda, 0)-C_{\ell, q}^{(k-1)}(\lambda, 0)$, which completes the proof of the first recurrence.

By applying the above proof to the case of poly-Cauchy polynomials with a $q$ parameter of the second kind together with using (1.4) for $\widehat{C}_{n, q}^{(k)}(\lambda, x)$ instead of $C_{n, q}^{(k)}(\lambda, x)$, one can obtain the second recurrence.

In the next result one finds the expressions for $\frac{d}{d x} C_{n, q}^{(k)}(\lambda, x)$ and $\frac{d}{d x} \widehat{C}_{n, q}^{(k)}(\lambda, x)$.

Theorem 3.3 For all $n \geq 0$,

$$
\frac{d}{d x} C_{n, q}^{(k)}(\lambda, x)=n ! \sum_{\ell=0}^{n-1} \frac{(-q)^{n-1-\ell}}{(n-\ell) \ell !} C_{\ell, q}^{(k)}(\lambda, x), \quad \frac{d}{d x} \widehat{C}_{n, q}^{(k)}(\lambda, x)=-n ! \sum_{\ell=0}^{n-1} \frac{(-q)^{n-1-\ell}}{(n-\ell) \ell !} \widehat{C}_{\ell, q}^{(k)}(\lambda, x) .
$$

Proof It is well known that $\frac{d}{d x} S_{n}(x)=\sum_{\ell=0}^{n-1}\left(\begin{array}{l}n \\ \ell\end{array}\right)\left\langle\bar{f}(t) \mid x^{n-\ell}\right\rangle S_{\ell}(x)$, where $S_{n}(x) \sim(g(t), f(t))$ and $\bar{f}(t)$ is the compositional inverse of $f(t)$ (see [14, 15]). In the present cases, see (1.4), one has either $\bar{f}(t)=\frac{1}{q} \log (1+q t)$ or $\bar{f}(t)=-\frac{1}{q} \log (1+q t)$. Note that $\left\langle\frac{1}{q} \log (1+q t) \mid x^{n-\ell}\right\rangle=$ $(-q)^{n-1-\ell}(n-1-\ell) !$. Thus, $\frac{d}{d x} C_{n, q}^{(k)}(\lambda, x)=n ! \sum_{\ell=0}^{n-1} \frac{(-q)^{n-1-\ell}}{(n-\ell) \ell !} C_{\ell, q}^{(k)}(\lambda, x)$ and $\frac{d}{d x} \widehat{C}_{n, q}^{(k)}(\lambda, x)=$ $-n ! \sum_{\ell=0}^{n-1} \frac{(-q)^{n-1-\ell}}{(n-\ell) !} \widehat{C}_{\ell, q}^{(k)}(\lambda, x)$, as required.

In the next theorem one uses the Korobov numbers. Recall that the Korobov numbers $K_{n}(\lambda)$ of the first kind are given by $\sum_{n \geq 0} K_{n}(\lambda) \frac{t^{n}}{n !}=\frac{\lambda t}{(1+t)^{\lambda}-1}$ (see [6, 7]).

Theorem 3.4 For all $n \geq 1$,

$$
\begin{aligned}
C_{n, q}^{(k)}(\lambda, x)= & x C_{n-1, q}^{(k)}(\lambda, x-q) \\
& +\frac{1}{n} \sum_{m=0}^{n} q^{n-m}\left(\begin{array}{c}
n \\
m
\end{array}\right) K_{n-m}(\lambda)\left(C_{m, q}^{(k-1)}(\lambda, x+(\lambda-1) q)-C_{m, q}^{(k)}(\lambda, x+(\lambda-1) q)\right),
\end{aligned}
$$




$$
\begin{aligned}
\widehat{C}_{n, q}^{(k)}(\lambda, x)= & -x \widehat{C}_{n-1, q}^{(k)}(\lambda, x+q) \\
& +\frac{1}{n} \sum_{m=0}^{n} q^{n-m}\left(\begin{array}{c}
n \\
m
\end{array}\right) K_{n-m}(\lambda)\left(\widehat{C}_{m, q}^{(k-1)}(\lambda, x-(\lambda-1) q)-C_{m, q}^{(k)}(\lambda, x-(\lambda-1) q)\right) .
\end{aligned}
$$

Proof Here only the proof of the first recurrence will be provided. Let $L_{k}=\operatorname{Lif}_{k}\left(\frac{(1+q t)^{\lambda}-1}{q \lambda}\right)$. By (1.2), we have $C_{n, q}^{(k)}(\lambda, y)=\left\langle L_{k}(1+q t)^{\frac{y}{q}} \mid x^{n}\right\rangle=A+B$, where $A=\left\langle L_{k} \frac{d}{d t}(1+q t)^{\frac{y}{q}} \mid x^{n-1}\right\rangle$ and $B=\left\langle\frac{d}{d t} L_{k}(1+q t)^{\frac{y}{q}} \mid x^{n-1}\right\rangle$. The term $A$ is given by $A=y\left\langle L_{k}(1+q t)^{\frac{y-q}{q}} \mid x^{n-1}\right\rangle=y C_{n-1, q}^{(k)}(\lambda, y-q)$. By (3.1), the term $B$ is given by

$$
\begin{aligned}
B & =\left\langle\frac{\lambda q t}{(1+q t)^{\lambda}-1}(1+q t)^{\frac{y+(\lambda-1) q}{q}} \frac{L_{k-1}-L_{k}}{t} \mid x^{n-1}\right\rangle \\
& =\frac{1}{n}\left\langle\frac{\lambda q t}{(1+q t)^{\lambda}-1}(1+q t)^{\frac{y+(\lambda-1) q}{q}}\left(L_{k-1}-L_{k}\right) \mid x^{n}\right\rangle .
\end{aligned}
$$

Note that the order of $L_{k-1}-L_{k}$ is at least one. Thus,

$$
\begin{aligned}
B & =\frac{1}{n}\left\langle\frac{\lambda q t}{(1+q t)^{\lambda}-1}(1+q t)^{\frac{y+(\lambda-1) q}{q}} L_{k-1} \mid x^{n}\right\rangle-\frac{1}{n}\left\langle\frac{\lambda q t}{(1+q t)^{\lambda}-1}(1+q t)^{\frac{y+(\lambda-1) q}{q}} L_{k} \mid x^{n}\right\rangle \\
& =\frac{1}{n}\left\langle\frac{\lambda q t}{(1+q t)^{\lambda}-1} \sum_{m=0}^{n}\left(C_{m, q}^{(k-1)}(\lambda, y+(\lambda-1) q)-C_{m, q}^{(k)}(\lambda, y+(\lambda-1) q)\right) \frac{t^{m}}{m !} \mid x^{n}\right\rangle \\
& =\frac{1}{n} \sum_{m=0}^{n}\left(\begin{array}{c}
n \\
m
\end{array}\right)\left(C_{m, q}^{(k-1)}(\lambda, y+(\lambda-1) q)-C_{m, q}^{(k)}(\lambda, y+(\lambda-1) q)\right)\left\langle\frac{\lambda q t}{(1+q t)^{\lambda}-1} \mid x^{n-m}\right\rangle .
\end{aligned}
$$

Thus, by expressing the Korobov numbers of the first kind, one obtains

$$
B=\frac{1}{n} \sum_{m=0}^{n}\left(\begin{array}{l}
n \\
m
\end{array}\right)\left(C_{m, q}^{(k-1)}(\lambda, y+(\lambda-1) q)-C_{m, q}^{(k)}(\lambda, y+(\lambda-1) q)\right) K_{n-m}(\lambda) q^{n-m} .
$$

Hence,

$$
\begin{aligned}
C_{n, q}^{(k)}(\lambda, y)= & y C_{n-1, q}^{(k)}(\lambda, y-q) \\
& +\frac{1}{n} \sum_{m=0}^{n} q^{n-m}\left(\begin{array}{c}
n \\
m
\end{array}\right) K_{n-m}(\lambda)\left(C_{m, q}^{(k-1)}(\lambda, y+(\lambda-1) q)-C_{m, q}^{(k)}(\lambda, y+(\lambda-1) q)\right),
\end{aligned}
$$

as required.

\section{Connections with families of polynomials}

Now, a few examples are presented on the connections with known families of polynomials. To do that, one uses the following fact from [14, 15]: For $s_{n}(x) \sim(g(t), f(t))$ and $r_{n}(x) \sim(h(t), \ell(t))$, let $s_{n}(x)=\sum_{k=0}^{n} c_{n, k} r_{k}(x)$. Then we have

$$
c_{n, k}=\frac{1}{k !}\left\langle\frac{h(\bar{f}(t))}{g(\bar{f}(t))}(\ell(\bar{f}(t)))^{k} \mid x^{n}\right\rangle .
$$


Let us start with the connection to Bernoulli polynomials $B_{n}^{(s)}(x)$ of order s. In the next result, one expresses the degenerate poly-Cauchy polynomials with a $q$ parameter in terms of Bernoulli polynomials of order s.

As analogs of (1.2) and (1.3), one defines the numbers $\mathbb{C}_{n, q}^{(s)}$ and $\widehat{\mathbb{C}}_{n, q}^{(s)}$ as $\left(\frac{q\left((1+q t)^{\frac{1}{q}}-1\right)}{\log (1+q t)}\right)^{s}=$ $\sum_{m \geq 0} \mathbb{C}_{n, q}^{(s)} \frac{t^{n}}{n !}$ and $\left(\frac{q\left(1-(1+q t)^{-\frac{1}{q}}\right)}{\log (1+q t)}\right)^{s}=\sum_{m \geq 0} \widehat{\mathbb{C}}_{n, q}^{(s)} \frac{t^{n}}{n !}$.

Theorem 4.1 For all $n \geq 0$,

$$
\begin{aligned}
& C_{n, q}^{(k)}(\lambda, x)=\sum_{m=0}^{n}\left(\sum_{\ell=m}^{n} \sum_{j=0}^{n-\ell}\left(\begin{array}{c}
n \\
\ell
\end{array}\right)\left(\begin{array}{c}
n-\ell \\
j
\end{array}\right) q^{\ell-m} S_{1}(\ell, m) C_{j, q}^{(k)}(\lambda, 0) \mathbb{C}_{n-\ell-j, q}^{(s)}\right) B_{m}^{(s)}(x), \\
& \widehat{C}_{n, q}^{(k)}(\lambda, x)=\sum_{m=0}^{n}\left((-1)^{m} \sum_{\ell=m}^{n} \sum_{j=0}^{n-\ell}\left(\begin{array}{c}
n \\
\ell
\end{array}\right)\left(\begin{array}{c}
n-\ell \\
j
\end{array}\right) q^{\ell-m} S_{1}(\ell, m) \widehat{C}_{j, q}^{(k)}(\lambda, 0) \widehat{\mathbb{C}}_{n-\ell-j, q}^{(s)}\right) B_{m}^{(s)}(x) .
\end{aligned}
$$

Proof Due to the similarity between the degenerate poly-Cauchy polynomials with a $q$ parameter of the first kind and of the second kind, only the proof details of the first identity will be provided, where the proof details of the second one are omitted. Let $C_{n, q}^{(k)}(\lambda, x)=$ $\sum_{m=0}^{n} c_{n, m} B_{m}^{(s)}(x)$. Then by (1.4), (4.1) and (2.6), one obtains

$$
c_{n, m}=\frac{1}{m !}\left\langle\left(\frac{q\left((1+q t)^{\frac{1}{q}}-1\right)}{\log (1+q t)}\right)^{s} \operatorname{Lif}_{k}\left(\frac{(1+q t)^{\lambda}-1}{q \lambda}\right) \mid\left(\frac{1}{q} \log (1+q t)\right)^{m} x^{n}\right\rangle,
$$

which, by (2.1) and (1.2), implies

$$
\begin{aligned}
c_{n, m} & =\frac{1}{q^{m}} \sum_{\ell=m}^{n}\left(\begin{array}{l}
n \\
\ell
\end{array}\right) q^{\ell} S_{1}(\ell, m)\left\langle\left(\frac{q\left((1+q t)^{\frac{1}{q}}-1\right)}{\log (1+q t)}\right)^{s} \mid \operatorname{Lif}_{k}\left(\frac{(1+q t)^{\lambda}-1}{q \lambda}\right) x^{n-\ell}\right\rangle \\
& =\frac{1}{q^{m}} \sum_{\ell=m}^{n}\left(\begin{array}{l}
n \\
\ell
\end{array}\right) q^{\ell} S_{1}(\ell, m)\left\langle\left(\frac{q\left((1+q t)^{\frac{1}{q}}-1\right)}{\log (1+q t)}\right)^{s} \mid \sum_{j=0}^{n-\ell} C_{j, q}^{(k)}(\lambda, 0) \frac{t^{j}}{j !} x^{n-\ell}\right\rangle \\
& =\frac{1}{q^{m}} \sum_{\ell=m}^{n} \sum_{j=0}^{n-\ell}\left(\begin{array}{l}
n \\
\ell
\end{array}\right)\left(\begin{array}{c}
n-\ell \\
j
\end{array}\right) q^{\ell} S_{1}(\ell, m) C_{j, q}^{(k)}(\lambda, 0)\left\langle\left(\frac{q\left((1+q t)^{\frac{1}{q}}-1\right)}{\log (1+q t)}\right)^{s} \mid x^{n-\ell-j}\right\rangle,
\end{aligned}
$$

which implies $c_{n, m}=\sum_{\ell=m}^{n} \sum_{j=0}^{n-\ell}\left(\begin{array}{c}n \\ \ell\end{array}\right)\left(\begin{array}{c}n-\ell \\ j\end{array}\right) q^{\ell-m} S_{1}(\ell, m) C_{j, q}^{(k)}(\lambda, 0) \mathbb{C}_{n-\ell-j, q}^{(s)}$, as required.

Using similar techniques to the proof of the previous theorem, one can express the degenerate poly-Cauchy polynomials in terms of other families, for instance, FrobeniusEuler polynomials (the proof is left to the interested reader). Note that the FrobeniusEuler polynomials $H_{n}^{(s)}(x \mid \mu)$ of order $s$ are defined by the generating function $\left(\frac{1-\mu}{e^{t}-\mu}\right)^{s} e^{x t}=$ $\sum_{n \geq 0} H_{n}^{(s)}(x \mid \mu) \frac{t^{n}}{n !}(\mu \neq 1)$, or equivalently, $H_{n}^{(s)}(x \mid \mu) \sim\left(\left(\frac{e^{t}-\mu}{1-\mu}\right)^{s}, t\right)$ (see [29, 30, 33, 34]).

Theorem 4.2 For all $n \geq 0$,

$$
\begin{aligned}
C_{n, q}^{(k)}(\lambda, x)= & \sum_{m=0}^{n}\left(\frac{\mu^{s}}{(1-\mu)^{s}} \sum_{\ell=m}^{n} \sum_{j=0}^{n-\ell} \sum_{i=0}^{s} \frac{\left(\begin{array}{c}
n \\
\ell
\end{array}\right)\left(\begin{array}{c}
n-\ell \\
j
\end{array}\right)\left(\begin{array}{l}
s \\
i
\end{array}\right) q^{\ell-m}}{(-\mu)^{i}} S_{1}(\ell, m)(i \mid q)_{n-\ell-j} C_{j, q}^{(k)}(\lambda, 0)\right) \\
& \times H_{m}^{(s)}(x \mid \mu),
\end{aligned}
$$




$$
\begin{aligned}
\widehat{C}_{n, q}^{(k)}(\lambda, x)= & \sum_{m=0}^{n}\left(\frac{\mu^{s}}{(\mu-1)^{s}} \sum_{\ell=m}^{n} \sum_{j=0}^{n-\ell} \sum_{i=0}^{s} \frac{(-1)^{m}\left(\begin{array}{c}
n \\
\ell
\end{array}\right)\left(\begin{array}{c}
n-\ell \\
j
\end{array}\right)\left(\begin{array}{l}
s \\
i
\end{array}\right) q^{\ell-m}}{(-\mu)^{i}}\right. \\
& \left.\times S_{1}(\ell, m)(-i \mid q)_{n-\ell-j} \widehat{C}_{j, q}^{(k)}(\lambda, 0)\right) H_{m}^{(s)}(x \mid \mu) .
\end{aligned}
$$

As another example, one can express our degenerate poly-Cauchy polynomials in terms of the rising factorials $(x \mid q)^{(m)}=x(x+q) \cdots(x+(m-1) q)$, as follows. Using the fact that $(x \mid q)^{(n)} \sim\left(1, \frac{1-e^{-q t}}{q}\right)$ with (1.2), (1.3), and (4.1), one obtains the following result.

Theorem 4.3 For all $n \geq 0$,

$$
\begin{aligned}
& C_{n, q}^{(k)}(\lambda, x)=\sum_{m=0}^{n}\left(\begin{array}{c}
n \\
m
\end{array}\right) C_{n-m, q}^{(k)}(\lambda,-q m)(x \mid q)^{(m)}, \\
& \widehat{C}_{n, q}^{(k)}(\lambda, x)=\sum_{m=0}^{n}(-1)^{m}\left(\begin{array}{l}
n \\
m
\end{array}\right) \widehat{C}_{n-m, q}^{(k)}(\lambda, 0)(x \mid q)^{(m)} .
\end{aligned}
$$

\section{Competing interests}

The authors declare that they have no competing interests.

\section{Authors' contributions}

All authors contributed equally and significantly in writing this article. All authors read and approved the final manuscript.

\section{Author details}

'Department of Mathematics, Sogang University, Seoul, 121-742, S. Korea. ${ }^{2}$ Department of Mathematics, Tianjin Polytechnic University, Tianjin, China. ${ }^{3}$ Department of Mathematics, Kwangwoon University, Seoul, 139-701, S. Korea. ${ }^{4}$ Institute of Natural Sciences, Far Eastern Federal University, Vladivostok, 690950, Russia.

\section{Acknowledgements}

The second author was appointed as a chair professor at Tianjin polytechnic University by Tianjin city in China from August 2015 to August 2019. We would like to express our gratitude to Professor Toufik Mansour for his comments and improvements. Our thanks also go to the referees for their comments and suggestions, which improved the present paper greatly. The work reported in this paper was conducted during the sabbatical year of Kwangwoon University in 2014.

Received: 9 June 2015 Accepted: 9 November 2015 Published online: 18 November 2015

\section{References}

1. Komatsu, T: Poly-Cauchy numbers with a q-parameter. Ramanujan J. 31, 353-371 (2013)

2. Dolgy, DV, Kim, DS, Kim, T, Mansour, T: Degenerate poly-Cauchy polynomials. Appl. Math. Comput. 269, 637-646 (2015)

3. Carlitz, L: A degenerate Staudt-Clausen theorem. Arch. Math. 7, 28-33 (1956)

4. Carlitz, L: Degenerate Stirling, Bernoulli and Eulerian numbers. Util. Math. 15, 51-88 (1979)

5. Ustinov, AV: Korobov polynomials and umbral analysis. Chebyshevskiі Sb. 4(4(8)), 137-152 (2003)

6. Korobov, NM: Special polynomials and their applications. Diophantine approximations. Math. Notes 2, 77-89 (1996)

7. Korobov, NM: On some properties of special polynomials. In: Proceedings of the IV International Conference 'Modern Problems of Number Theory and Its Applications', Tula, 2001, vol. 1, pp. 40-49 (2001) (in Russian)

8. Kim, DS, Kim, T: Some identities of degenerate Euler polynomials arising from $p$-adic fermionic integrals on $\mathbb{Z}_{p}$. Integral Transforms Spec. Funct. 26(4), 295-302 (2015)

9. Kim, DS, Kim, T: A note on degenerate poly-Bernoulli numbers and polynomials. Adv. Differ. Equ. 2015, 258 (2015)

10. Kim, DS, Kim, T: Some identities of degenerate special polynomials. Open Math. 13, 380-389 (2015)

11. Kim, DS, Kim, T, Dolgy, DV: A note on degenerate Bernoulli numbers and polynomials associated with $p$-adic invariant integral on $\mathbb{Z}_{p}$. Appl. Math. Comput. 259, 198-204 (2015)

12. Kim, DS, Kim, T, Dolgy, DV: Degenerate $q$-Euler polynomials. Adv. Differ. Equ. 2015, 246 (2015)

13. Kim, DS, Kim, T, Kwon, HI, Mansour, T: Degenerate poly-Bernoulli polynomials with umbral calculus viewpoint. J. Inequal. Appl. 2015, 228 (2015)

14. Roman, S: More on the umbral calculus, with emphasis on the q-umbral calculus. J. Math. Anal. Appl. 107, 222-254 (1985)

15. Roman, S: The Umbral Calculus. Dover, New York (2005)

16. Agapito, J: Riordan arrays from an umbral symbolic viewpoint. Bol. Soc. Port. Mat. 5-8 (2012) (special issue)

17. Araci, S: Novel identities involving Genocchi numbers and polynomials arising from applications of umbral calculus. Appl. Math. Comput. 233, 599-607 (2014) 
18. Biedenharn, LC, Gustafson, RA, Lohe, MA, Louck, JD, Milne, SC: Special functions and group theory in theoretical physics. In: Special Functions: Group Theoretical Aspects and Applications. Math. Appl., pp. 129-162. Reidel, Dordrecht (1984)

19. Di Bucchianico, A, Loeb, D: A selected survey of umbral calculus. Electron. J. Comb. 2, DS3 (2000)

20. Dattoli, G, Levi, D, Winternitz, P: Heisenberg algebra, umbral calculus and orthogonal polynomials. J. Math. Phys. 49(5), 053509 (2008)

21. Sen, E: Theorems on Apostol-Euler polynomials of higher order arising from Euler basis. Adv. Stud. Contemp. Math. (Kyungshang) 23(2), 337-345, 433-438 (2013)

22. Kim, DS, Kim, T: Higher-order Cauchy of the first kind and poly-Cauchy of the first kind mixed type polynomials. Adv. Stud. Contemp. Math. (Kyungshang) 23(4), 621-636 (2013)

23. Araci, $\mathrm{S}, \mathrm{Acikgoz}, \mathrm{M}$, Kilicman, A: Extended $p$-adic $q$-invariant integrals on $\mathbb{Z}_{p}$ associated with applications of umbral calculus. Adv. Differ. Equ. 2013, 96 (2013)

24. Qi, F, Wang, T: Umbral calculus and invariant sequences. Ars Comb. 101, 257-264 (2011)

25. Kim, T, Mansour, T: Umbral calculus associated with Frobenius-type Eulerian polynomials. Russ. J. Math. Phys. 21(4), 484-493 (2014)

26. Wilson, BG, Rogers, FG: Umbral calculus and the theory of multispecies nonideal gases. Physica A 139, 359-386 (1986)

27. Kim, DS, Kim, T: Poly-Cauchy numbers and polynomials of the second kind with umbral calculus viewpoint. Adv. Differ. Equ. 2014, 36 (2014)

28. Gasper, G, Rahman, M: Basic Hypergeometric Series. With a foreword by Richard Askey. Encyclopedia of Mathematics and Its Applications, vol. 35. Cambridge University Press, Cambridge (1990)

29. Bayad, A, Kim, T: Identities involving values of Bernstein, $q$-Bernoulli, and $q$-Euler polynomials. Russ. J. Math. Phys. 18(2), 133-143 (2011)

30. Ding, D, Yang, J: Some identities related to the Apostol-Euler and Apostol-Bernoulli polynomials. Adv. Stud. Contemp Math. 20(1), 7-21 (2010)

31. Kim, DS, Kim, T: A note on poly-Bernoulli and higher-order poly-Bernoulli polynomials. Russ. J. Math. Phys. 22, 26-33 (2015)

32. Kim, DS, Kim, T, Lee, S-H, Rim, S-H: Umbral calculus and Euler polynomials. Ars Comb. 112, 293-306 (2013)

33. Araci, S, Acikgoz, M: A note on the Frobenius-Euler numbers and polynomials associated with Bernstein polynomials. Adv. Stud. Contemp. Math. 22(3), 399-406 (2012)

34. Kim, T: Identities involving Laguerre polynomials derived from umbral calculus. Russ. J. Math. Phys. 21(1), 36-45 (2014)

\section{Submit your manuscript to a SpringerOpen ${ }^{\odot}$ journal and benefit from:}

- Convenient online submission

- Rigorous peer review

Immediate publication on acceptance

- Open access: articles freely available online

- High visibility within the field

- Retaining the copyright to your article 